\title{
Narrow-Banding Images and Structures of Microvessels of Colonic Lesions
}

\author{
Ken-ichi Mizuno $\cdot$ Shin-ei Kudo $\cdot$ Kazuo Ohtsuka \\ Shigeharu Hamatani $\cdot$ Yoshiki Wada $\cdot$ \\ Haruhiro Inoue $\cdot$ Yutaka Aoyagi
}

Received: 17 August 2010/Accepted: 1 December 2010/Published online: 28 December 2010

(C) The Author(s) 2010. This article is published with open access at Springerlink.com

\begin{abstract}
Background Magnifying colonoscopy with NBI has been shown to be useful for the differential diagnosis of tumors. However, the relationship between findings on NBI magnification and the microvessel architecture of colorectal lesions remains to be clarified.

Aims The aim of this study was to clarify the correlation between NBI findings and the microvascular architecture of colorectal lesions according to the depth of microvessels from the mucosal surface.

Methods A total of 22 colorectal lesions (11 tubular adenomas and 11 hyperplasia) obtained from 22 patients were studied. These lesions were analyzed microscopically on tissue specimens immunostained with CD34. Threedimensional images were reconstructed from serial sections of tubular adenomas, hyperplasia, and normal mucosa.

Results Three-dimensional reconstructed images of tubular adenoma and normal mucosa to a depth of less than $150 \mu \mathrm{m}$ from the mucosal surface showed similar structures to images obtained by NBI magnification. Microvessel diameter was significantly larger in tubular adenoma than in normal mucosa $(P=0.002)$ and hyperplasia
\end{abstract}

K. Mizuno $(\bowtie) \cdot$ S. Kudo $\cdot$ K. Ohtsuka $\cdot$ S. Hamatani ·

$\mathrm{Y}$. Wada $\cdot \mathrm{H}$. Inoue

Digestive Disease Center, Showa University Northern

Yokohama Hospital, 35-1, Chigasaki-chuo, Tsuduki-ku,

Yokohama-city 224-8503, Japan

e-mail: kenken_mjp@yahoo.co.jp

\section{Y. Aoyagi}

Department of Gastroenterology and Hepatology,

Graduate School of Medical and Dental Sciences,

Niigata University, 1-757 Asahimachi-dori, Chuo-ku,

Niigata, Niigata 951-8122, Japan
$(P=0.034)$, and microvessel area was significantly larger in tubular adenoma than in normal mucosa $(P<0.001)$ and hyperplasia $(P<0.001)$ only in the superficial mucosal layer (to a depth of less than $150 \mu \mathrm{m}$ ).

Conclusions TA was characterized by thicker microvessels and higher volume of microvessels than NM and HP. Compared with white light, NBI can more accurately depict the characteristics of microvessels because it uses light with short wavelengths, thereby contributing to high diagnostic capability.

Keywords Colorectal neoplasms .

Computer-assisted 3-dimensional imaging ·

Narrow-band imaging · Colonoscopy

\section{Introduction}

To reduce morbidity and mortality from colorectal cancer, colonoscopy is considered the method of choice for the detection and removal of colon neoplasms, especially adenomatous lesions and early cancers. Distinguishing colorectal neoplasms from non-neoplastic lesions is one of the main roles of colonoscopy.

Narrow-band imaging (NBI) is a newly developed imaging technique that uses special filters to narrow the bandwidth of light [1]. NBI systems allow visualization of microvessels and the surface structure of the mucosa. NBI in combination with magnifying endoscopy has been reported to be useful for the diagnosis of neoplasms arising in the esophagus, stomach, and oropharynx [2-5]. Several studies have shown that NBI with magnifying endoscopy (NBI magnification) is useful for differentiating hyperplastic from neoplastic lesions in the colon. Machida and others have reported that NBI is superior to conventional 
colonoscopy and equivalent to chromoendoscopy for differentiating hyperplastic from neoplastic polyps [6-18]. However, the relationship between findings on NBI magnification and the microvessel architecture of colorectal lesions and the reasons why NBI magnification is useful for differentiating hyperplastic from neoplastic polyps remain to be clarified.

In the present study, images obtained by NBI magnification were compared with computer-aided 3-dimensional reconstructed images. Three-dimensional visualization of the microvessel network according to the distance from mucosal surface is an effective tool for understanding the depth of microvessels visualized on NBI magnification. Next, we quantitatively analyzed three vascular variables (diameter, area, number) in hyperplasia (HP), tubular adenoma (TA), and normal mucosa (NM) according to the depth from the mucosal surface.

\section{Materials and Methods}

\section{Patients}

A total of 22 colorectal lesions from 22 patients were studied. All patients underwent colonoscopy at the Digestive Disease Center, Showa University Northern Yokohama Hospital. The subjects were 14 men and 8 women with a mean age of 56.7 years (range 35-79). All patients received a detailed explanation of the study protocol and gave written informed consent.

\section{Colonoscopy Procedure and Lesions}

All colonoscopic examinations were performed by experienced endoscopists in our center, using a magnifying videoendoscope (CF-H260AZI; Olympus, Tokyo, Japan) and a standard videoendoscopic system (LUCERA; Olympus) equipped with the standard optical filter (white light) and the NBI system. All lesions were examined by NBI magnification. NBI diagnoses were based on the Wada classification [15]. All lesions were examined by NBI magnification. NBI diagnoses were based on the Wada classification. All lesions were resected endoscopically and fixed in $10 \%$ buffered formalin for 12-48 h. Hematoxylineosin-stained sections were histopathologically examined by a single pathologist in our hospital. The histopathological diagnosis was established according to the World Health Organization criteria. The 22 lesions analyzed in this study were $11 \mathrm{HP}$ lesions and $11 \mathrm{TA}$. NBI diagnosis and histopathological diagnosis were consistent in all lesions. All HP lesions were similar in color to the adjacent normal mucosa on NBI without magnification, and all had an unclear vessel network, even on NBI magnification.
This corresponded to the faint pattern of the Wada classification. The mean size of TA was $5.3 \pm 2.4 \mathrm{~mm}$. All TA appeared brownish on NBI without magnification and had a regular meshwork of smooth microvessels on NBI magnification. This corresponded to the regular network pattern of the Wada classification. The mean size of HP was $7.3 \pm 2.6 \mathrm{~mm}$.

\section{Microvessel Immunohistochemical Staining}

All 22 lesions were examined immunohistochemically by CD34 staining of endothelial cells. The histopathological characteristics of the tumors were reviewed, and the most representative tumor block was selected for immunohistochemical analysis. Sections $(4 \mu \mathrm{m}$ thick) were cut from formalin-fixed and paraffin-embedded blocks of tumor tissue. Anti-CD34 monoclonal antibody (Nichirei, Tokyo, Japan) and the streptavidin-biotin method (Dako LSAB kit; Dako Japan, Kyoto, Japan) were used for immunohistochemical staining.

\section{Three-Dimensional Reconstruction}

For 3-dimensional reconstruction, we prepared 100 serial sections of NM (3.5 $\mu \mathrm{m}$ thick), HP (3.5 $\mu \mathrm{m}$ thick), and TA ( $5 \mu \mathrm{m}$ thick) by CD34 immunostaining. The microscope images of serial sections were digitized and recorded. The outlines of the vessels were traced and registered for each section, and 3-dimensional reconstructed images were produced using a computer reconstruction program (TRI/ 3D SRF II; RATOC System Engineering, Tokyo, Japan) [19].

\section{Microvessel Analysis}

One section immunostained with CD34 was analyzed per lesion. The entire tumor section was first carefully scanned at $\times 100(\times 10$ ocular, $\times 10$ objective $)$ to find the most vascular areas ("hotspots"). These hotspots were identified as areas with the highest density of brown-stained CD34 cells $[20,21]$. Any brown-stained endothelial cell or endothelial cell cluster that was clearly separated from the adjacent microvessels was considered a single countable vessel. Microscope images of magnified fields $(\times 200)$ within hotspots were digitized and recorded. Images were captured using an optical microscope connected to a color video camera and saved on a disc as bmp files. Each digitized image corresponded to a microscope field measuring $500 \mu \mathrm{m}^{2}(960 \times 960$ pixels $)$.

To evaluate microvessels, we counted the numbers of microvessels, measured the diameters and areas of microvessels, and measured the distance from each vessel to the mucosal surface. The minor axis of each microvessel was 
defined as the diameter of the vessel. The vertical distance from the center of the vessel to the mucosal surface (mucosal surface-vessel distance) was determined by counting the number of pixels on digitized images. To measure the microvessel area, outlines of all vessels were traced and painted black with the use of Microsoft paint software (Microsoft, Redmond, WA, USA). Subsequently, image-processing software (NIH Scion Image Beta 4.0.3; Scion, http://www.scioncorp.com/) was used to measure the area of each vessel as follows: image conversion to gray scale, thresholding, scale setting, and area measurement [22]. For each section of HP, microvessels of the NM that were distant from the hyperplastic lesion were measured.

To evaluate the correlation between microvessel-related variables and mucosal surface-vessel distance, microvessels were divided into the following three groups according to the mucosal surface-vessel distance: upper layer $(\leq 150 \mu \mathrm{m})$, middle layer $(>150$ to $\leq 300)$, and lower layer $(>300$ to $\leq 450)$. Three variables were analyzed in this study: microvessel diameter (MVD), the average diameter of all individual microvessels in each layer; microvessel area (MVA), the sum of the areas of all individual microvessels in each layer; and microvessel count (MVC), the sum of the number of all individual microvessels in a field. Data were evaluated by the Mann-Whitney test.

\section{Results}

Three-Dimensional Reconstruction

Figure 1 shows a compilation of CD34-stained sections (first row), images on NBI magnification (second row), and 3-dimensional reconstructed images of microvessels (third and fourth rows) for NM, HP, and TA. Three-dimensional images were reconstructed for microvessels to depths of less than $450 \mu \mathrm{m}$ and less than $150 \mu \mathrm{m}$ from the mucosal surface.

Reconstructed images of TA and NM to a depth of less than $450 \mu \mathrm{m}$ from the mucosal surface showed complicated structures because of many layers of microvessels. In contrast, reconstructed images of TA and NM to a depth of less than $150 \mu \mathrm{m}$ from the mucosal surface showed structures similar to those on NBI magnification.

Three-dimensional reconstructed images of the microvessels of NM showed a regular honeycomb-like network, similar to the images obtained by NBI magnification. Microvascular networks surrounded the openings of mucosal glands. Three-dimensional reconstruction of $\mathrm{HP}$ showed the absence of microvascular networks. The microvasculature was fragmentary. NBI magnification of HP showed an unclear vascular pattern. Three-dimensional reconstruction of TA showed vascular networks similar to
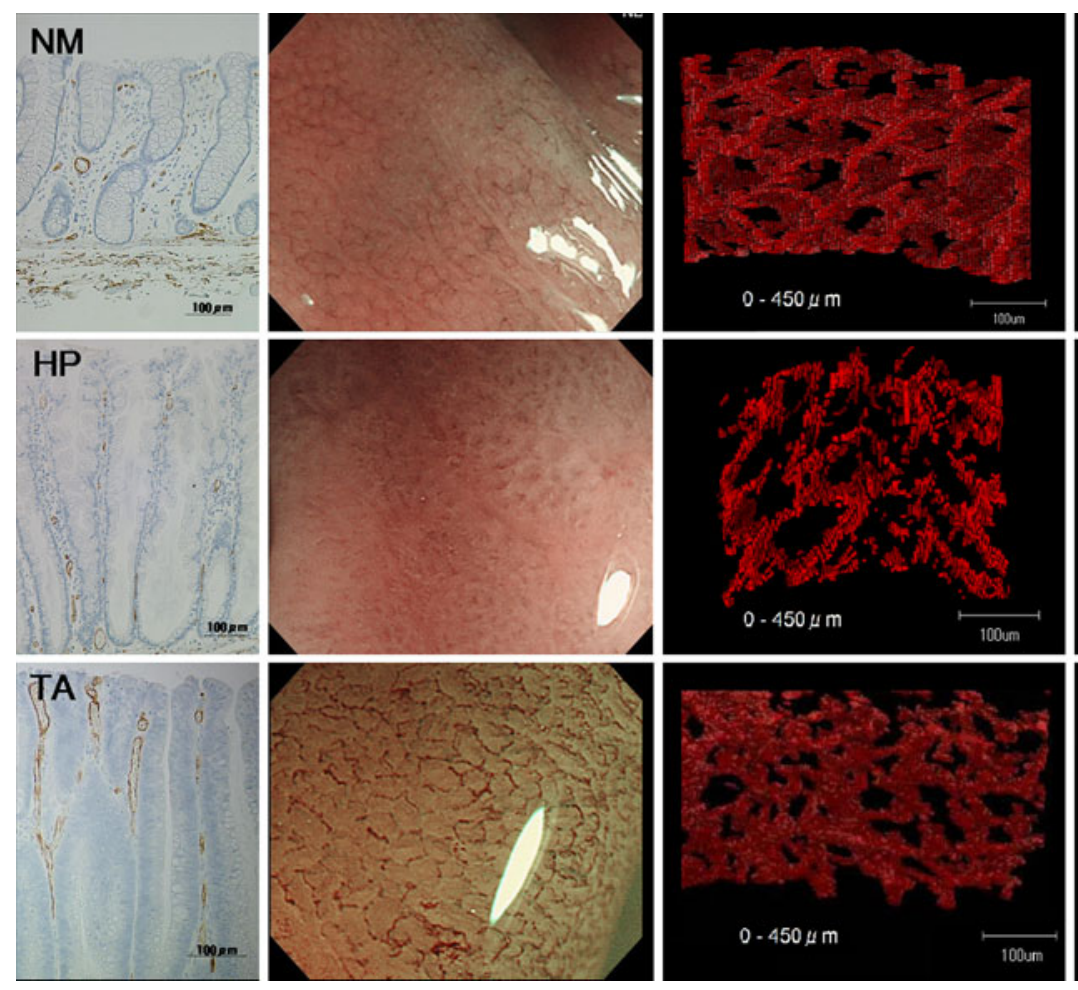
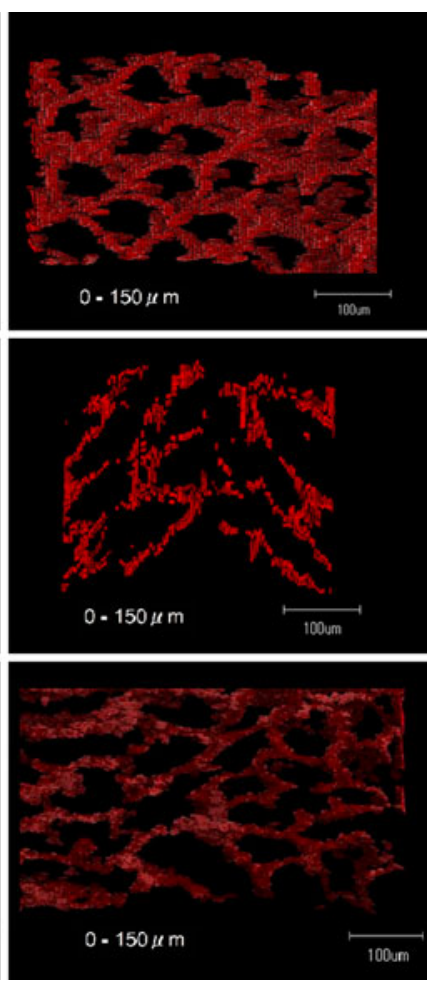

Fig. 1 CD34-stained sections (first row), images on NBI magnification (second row), and 3-dimensional reconstruction of microvessels (third and fourth rows) in normal mucosa (NM), hyperplasia (HP), and tubular adenoma (TA). Three-dimensional images were reconstructed for microvessels to depths of less than $450 \mu \mathrm{m}$ (third row) and less than $150 \mu \mathrm{m}$ (fourth row) from the mucosal surface 
Table 1 Relationship between microvessel diameter $(M V D)$ and mucosal surface-vessel distance

\begin{tabular}{lccc}
\hline & \multicolumn{2}{l}{ Average MVD $(\mu \mathrm{m})$} & \\
\cline { 2 - 4 } & Upper $(\leq 150)^{\mathrm{a}}$ & Middle $(>150 \text { to } \leq 300)^{\mathrm{a}}$ & Lower $(>300 \text { to } \leq 450)^{\mathrm{a}}$ \\
\hline Normal mucosa (NM) & $7.4 \pm 0.9$ & $6.9 \pm 1.5$ & $8.8 \pm 3.3$ \\
Hyperplasia (HP) & $7.6 \pm 2.1$ & $10.1 \pm 3.0$ & $8.7 \pm 1.8$ \\
Tubular adenoma (TA) & $10.9 \pm 3.4$ & $8.3 \pm 3.1$ & $7.6 \pm 1.3$ \\
\hline
\end{tabular}

Microvessel diameter was defined as the average diameter of all individual microvessels in each layer. MVD differed significantly between NM and TA $(P=0.002)$ and between HP and TA $(P=0.034)$ in the upper layer

${ }^{a}$ Microvessels were divided into the following three groups according to mucosal surface-vessel distance: upper layer $(\leq 150 \mu \mathrm{m})$, upper and middle layer ( $>0$ to $\leq 300 \mu \mathrm{m})$, and all layers $(>0$ to $\leq 450 \mu \mathrm{m})$

Table 2 Relationship between microvessel diameter $(M V D)$ and mucosal surface-vessel distance

\begin{tabular}{lccc}
\hline & \multicolumn{2}{c}{ Average MVD $(\mu \mathrm{m})$} & \\
\cline { 2 - 4 } & ${\text { Upper }(\leq 150)^{\mathrm{a}}}^{*}$ All $(>0 \text { to } \leq 450)^{\mathrm{a}}$ \\
\hline Normal mucosa (NM) & $7.4 \pm 0.9$ & $7.3 \pm 1.1$ & $7.6 \pm 1.5$ \\
Hyperplasia (HP) & $7.6 \pm 2.1$ & $8.8 \pm 1.8$ & $8.7 \pm 1.5$ \\
Tubular adenoma (TA) & $10.9 \pm 3.4$ & $10.3 \pm 3.1$ & $9.5 \pm 2.4$ \\
\hline
\end{tabular}

Microvessel diameter was defined as the average diameter of all individual microvessels in each layer. MVD differed significantly between HP and TA in the upper layer $(P=0.034)$, but there were no significant differences in upper + middle $(P=0.289)$ layer or all layers $(P=0.762)$

${ }^{a}$ Microvessels were divided into the following three groups according to mucosal surface-vessel distance: upper layer $(\leq 150 \mu \mathrm{m})$, upper and middle layer ( $>0$ to $\leq 300 \mu \mathrm{m})$, and all layers $(>0$ to $\leq 450 \mu \mathrm{m})$

those present in NM. However, the networks around the mucosal glands were elongated. Similar to NM, 3-dimensional reconstructed images of TA were consistent with the images obtained by NBI magnification. In contrast, HP showed a different pattern from that obtained by NBI magnification in the upper layer.

\section{Microvessel Analysis}

Mean MVD values are summarized in Tables 1 and 2 and Fig. 2. In the upper layer, MVD differed significantly between NM and TA $(P=0.002)$ and between HP and TA $(P=0.034)$, but did not differ significantly between NM and HP. In contrast, there were no significant differences in MVD between NM, HP, or TA in the middle or lower layers. Mean MVD was slightly but not significantly higher in TA than in HP when microvessels in all layers were compared or when the upper and middle layers were compared.

Mean MVA values are summarized in Tables 3 and 4 and Fig. 2. In the upper layer, MVA differed significantly between NM and TA $(P<0.001)$ as well as between HP and TA $(P<0.001)$, but did not differ significantly between NM and HP. There were no significant differences in MVA between NM, HP, or TA in the middle layer or lower layer.
Mean MVC values are summarized in Table 5 and Fig. 2. In the upper layer, MVC differed significantly between NM and HP $(P=0.04)$ and between HP and TA $(P=0.012)$, but did not differ between NM and TA. There were no significant differences in MVC between NM, HP, or TA in the middle layer or lower layer. MVC did not differ significantly between TA and HP in all layers or in the upper plus middle layers.

\section{Discussion}

NBI systems have two major characteristics. First, these systems use light with a wavelength of $415 \mu \mathrm{m}$, which is absorbed by hemoglobin, thereby enhancing the visualization of microvessels. Second, because NBI systems use light with short wavelengths the penetration depth is shallow. The structure of the superficial layer can therefore be visualized more clearly than that on imaging with the use of white light. Many studies have reported that NBI is useful for the diagnosis of colorectal lesions [9, 23, 24]. To our knowledge, however, no previous study has examined the relationship between NBI images and the microstructures of blood vessels according to the depth from the mucosal surface. A better understanding of this relationship may facilitate the diagnosis and evaluation of lesions. 

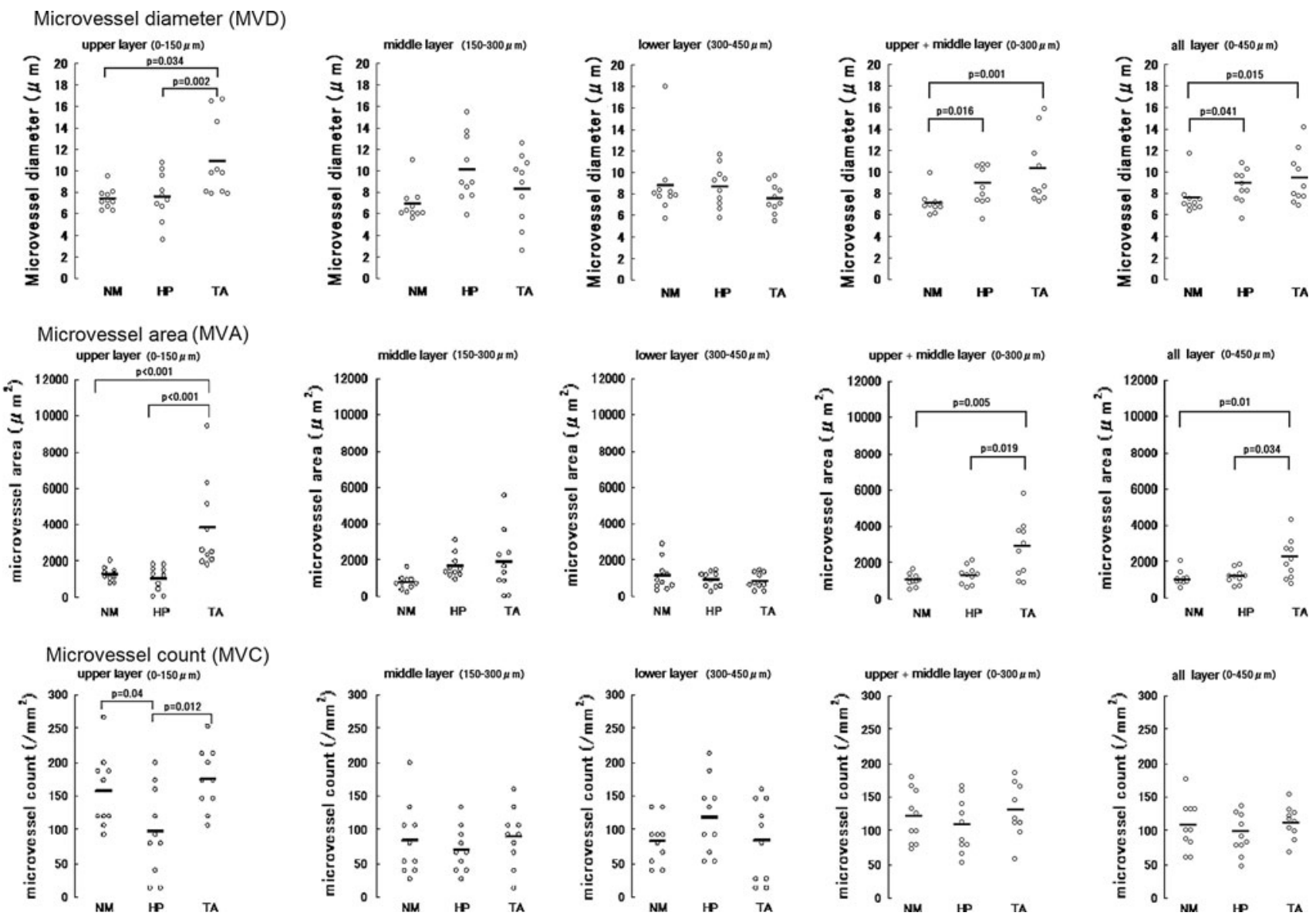

Fig. 2 MVD, MVA, and MVC of NM, HP, and TA. MVD and MVA differed significantly between NM and TA and between HP and TA, but did not differ significantly between NM and HP in the upper layer. MVC differed significantly between NM and HP and between TA and $\mathrm{HP}$ in the upper layer. There were no significant differences in MVD, MVA, or MVC between NM, HP, or TA in the middle layer or lower layer. When microvessel analysis was done after combining the upper and middle layers $(0-300 \mu \mathrm{m})$ or the upper, middle, and lower layers $(0-450 \mu \mathrm{m})$, MVD differed significantly between HP and TA in the upper layer, but there were no significant differences in upper and middle layer $(0-300 \mu \mathrm{m})$ or all layers $(0-450 \mu \mathrm{m})$

Table 3 Relationship between microvessel area $(M V A)$ and mucosal surface-vessel distance

\begin{tabular}{|c|c|c|c|}
\hline & \multicolumn{3}{|c|}{ Average MVA $\left(\mu \mathrm{m}^{2}\right)$} \\
\hline & Upper $(\leq 150)^{\mathrm{a}}$ & Middle $(>150 \text { to } \leq 300)^{\mathrm{a}}$ & Lower $(>300 \text { to } \leq 450)^{\mathrm{a}}$ \\
\hline Normal mucosa (NM) & $1,263 \pm 364$ & $757 \pm 365$ & $1,129 \pm 808$ \\
\hline Hyperplasia (HP) & $1,031 \pm 653$ & $1,651 \pm 637$ & $911 \pm 398$ \\
\hline Tubular adenoma (TA) & $3,782 \pm 2,361$ & $1,863 \pm 1,626$ & $845 \pm 420$ \\
\hline
\end{tabular}

Microvessel area was defined as the sum of the areas of all individual microvessels in each layer. MVA differed significantly between NM and TA $(P<0.001)$, as well as between HP and TA $(P<0.001)$ in the upper layer

${ }^{\text {a }}$ Microvessels were divided into the following three groups according to mucosal surface-vessel distance: upper layer $(\leq 150 \mu \mathrm{m})$, upper and middle layer $(>0$ to $\leq 300 \mu \mathrm{m})$, and all layers $(>0$ to $\leq 450 \mu \mathrm{m})$

First, three-dimensional images of TA, HP and NM were reconstructed for microvessels in this study. An important advantage of 3-dimensional reconstruction based on digitized images is that microvascular structures can be reconstructed from arbitrarily selected layers of lesions. This enables images obtained by NBI magnification to be compared with microvascular structure according to the distance from the mucosal surface. Reconstructed images of TA and NM to a depth of less than $150 \mu \mathrm{m}$ were in accord with the images obtained by NBI magnification. On the other hand, reconstructed images to a depth of less than $450 \mu \mathrm{m}$ were not consistent with NBI images because 
Table 4 Relationship between microvessel area $(M V A)$ and mucosal surface-vessel distance

\begin{tabular}{|c|c|c|c|}
\hline & \multicolumn{3}{|c|}{ Average MVA $\left(\mu \mathrm{m}^{2}\right)$} \\
\hline & Upper $(\leq 150)^{\mathrm{a}}$ & Upper + middle $(>0 \text { to } \leq 300)^{\mathrm{a}}$ & All $(>0 \text { to } \leq 450)^{\mathrm{a}}$ \\
\hline Normal mucosa (NM) & $1,263 \pm 364$ & $1,010 \pm 339$ & $1,050 \pm 413$ \\
\hline Hyperplasia (HP) & $1,031 \pm 653$ & $1,288 \pm 502$ & $1,162 \pm 405$ \\
\hline Tubular adenoma (TA) & $3,782 \pm 2,361$ & $2,823 \pm 1,601$ & $2,163 \pm 1,102$ \\
\hline
\end{tabular}

MVA differed significantly between NM and TA $(P<0.001)$, as well as between HP and TA in the upper layer $(P<0.001)$, upper + middle layer $(P=0.008)$, and all layers $(P=0.034)$

${ }^{\text {a }}$ Microvessels were divided into the following three groups according to mucosal surface-vessel distance: upper layer ( $\left.\leq 150 \mu \mathrm{m}\right)$, upper and middle layer $(>0$ to $\leq 300 \mu \mathrm{m})$, and all layers $(>0$ to $\leq 450 \mu \mathrm{m})$

Table 5 Relationship between microvessel count $(M V C)$ and mucosal surface-vessel distance

\begin{tabular}{lccc}
\hline & \multicolumn{2}{l}{ Average MVC (no. of microvessels/mm $\left.{ }^{2}\right)$} & Lower $(>300 \text { to } \leq 450)^{\mathrm{a}}$ \\
\cline { 2 - 4 } & Upper $(\leq 150)^{\mathrm{a}}$ & Middle $(>150 \text { to } \leq 300)^{\mathrm{a}}$ & $84.0 \pm 51.0$ \\
\hline Normal mucosa (NM) & $157.3 \pm 51.5$ & $82.7 \pm 32.0$ & $70.7 \pm 31.6$ \\
Hyperplasia (HP) & $97.3 \pm 62.3$ & $118.7 \pm 52.8$ & $84.0 \pm 56.6$ \\
Tubular adenoma (TA) & $89.3 \pm 40.5$ & $174.7 \pm 43.6$ & 2 \\
\hline
\end{tabular}

Microvessel count was defined as the sum of the number of all individual microvessels in a field. MVC differed significantly between NM and HP $(P=0.04)$ and between HP and TA $(P=0.012)$ in the upper layer

${ }^{a}$ Microvessels were divided into the following three groups according to mucosal surface-vessel distance: upper layer $(\leq 150 \mu \mathrm{m})$, upper and middle layer ( $>0$ to $\leq 300 \mu \mathrm{m})$, and all layers $(>0$ to $\leq 450 \mu \mathrm{m})$

many layers of microvessels appeared to overlap in a complex fashion.

Although we could not accurately evaluate the depth of microvessels seen on NBI on the basis of 3-dimensional reconstructed images, these results suggested that NBI magnification can accurately depict superficial microvessels in mucosa located to a depth of around $150 \mu \mathrm{m}$.

When microvessel analysis was performed according to depth, MVD, MVA, and MVC were found to differ significantly among NM, HP, and TA only in the upper layer. When microvessel analysis was done after combining the upper and middle layers $(0-300 \mu \mathrm{m})$ or the upper, middle, and lower layers $(0-450 \mu \mathrm{m})$, mean MVD was slightly but not significantly higher in TA than in HP. Moreover, the statistical significance of the difference in MVA between TA and HP (i.e., MVA greater in TA than in HP) became stronger when only the most superficial layers were evaluated.

Several investigators have reported that the diameter of microvessels is larger in neoplastic lesions than in nonneoplastic lesions [9, 23-25]. Therefore, the fine microvascular architecture of TA is often evaluated by means of NBI magnification.

Our results were consistent with the findings of previous studies. In our study, MVD tended to be larger in TA than in HP in the more superficial layers. However, MVD differed significantly between TA and HP only in the upper layer. Because NBI uses light with shorter wavelengths than white light, it can more clearly depict microvessels in the superficial layer of the mucosa. One factor underlying the high diagnostic capability of NBI magnification is the fact that NBI can more accurately detect differences in MVD between TA and HP (i.e., MVD is larger for TA than HP) in the superficial layer, as compared with techniques using white light.

Several investigators have reported that TA lesions can be discriminated from non-neoplastic lesions on NBI because the surface of adenomas is darker brown than that of non-neoplastic lesions [11]. In our study, the MVA of microvessels differed significantly between TA and nonneoplastic lesions. The difference in MVA was particularly large in the superficial layer. MVA is thought to correlate with the volume of microvessels. TAs thus had more microvessels than non-neoplastic lesions in the superficial layer. This higher microvessel volume is considered responsible for the darker brown appearance of adenomas on NBI.

MVD and MVA differed significantly between TA and non-neoplastic lesions, whereas MVC did not. It is speculated that thicker and higher volumes of microvessels are important characteristics of TA.

Tanaka et al. [26] reported that some HP lesions had features similar to those of TA. In our study, some HP lesions had large MVD and MVA, as shown in Fig. 1. Such HP lesions may be difficult to distinguish from TA. 
Our study had a limitation. We assessed microvessels to a depth of only $450 \mu \mathrm{m}$. However, 3-dimensional reconstructed images of microvessels to a depth of $150 \mu \mathrm{m}$ were consistent with the images on NBI. Therefore, a depth down to $450 \mu \mathrm{m}$ was considered a sufficient range for evaluating microvessels in the superficial layer of the mucosa.

In conclusion, our results suggest that TA is characterized by thicker microvessels and a higher volume of microvessels than HP and NM. This tendency was most clearly demonstrated in the superficial layer of the mucosa. Because NBI using light with shorter wavelengths than that of white light, it can more reliably detect the characteristics of microvessels, potentially contributing to higher diagnostic accuracy.

Acknowledgments This work was supported in part by a Grant for Cancer Research from the Ministry of Health, Labor and Welfare Ministry of Japan, and Core Research for Evolutional Science and Technology (CREST) from the Japan Science and Technology Agency (JST).

Open Access This article is distributed under the terms of the Creative Commons Attribution Noncommercial License which permits any noncommercial use, distribution, and reproduction in any medium, provided the original author(s) and source are credited.

\section{References}

1. Gono K, Obi T, Yamaguchi M, et al. Appearance of enhanced tissue features in narrow-band endoscopic imaging. J Biomed Opt. 2004;9:568-577.

2. Inoue H, Sasajima K, Kaga M, et al. Endoscopic in vivo evaluation of tissue atypia in the esophagus using a newly designed integrated endocytoscope: a pilot trial. Endoscopy. 2006;38: 891-895.

3. Hamamoto Y, Endo T, Nosho K, et al. Usefulness of narrow-band imaging endoscopy for diagnosis of Barrett's esophagus. J Gastroenterol. 2004;39:14-20.

4. Nakayoshi T, Tajiri H, Matsuda K, et al. Magnifying endoscopy combined with narrow band imaging system for early gastric cancer: correlation of vascular pattern with histopathology (including video). Endoscopy. 2004;36:1080-1084.

5. Muto M, Katada C, Sano Y, Yoshida S. Narrow band imaging: a new diagnostic approach to visualize angiogenesis in superficial neoplasia. Clin Gastroenterol Hepatol. 2005;3:S16-S20.

6. Kudo S, Rubio CA, Teixeira CR, Kashida H, Kogure E. Pit pattern in colorectal neoplasia: endoscopic magnifying view. Endoscopy. 2001;33:367-373.

7. Machida H, Sano Y, Hamamoto Y, et al. Narrow-band imaging in the diagnosis of colorectal mucosal lesions: a pilot study. Endoscopy. 2004;36:1094-1098.

8. Tischendorf JJ, Wasmuth HE, Koch A, et al. Value of magnifying chromoendoscopy and narrow band imaging (NBI) in classifying colorectal polyps: a prospective controlled study. Endoscopy. 2007;39:1092-1096.
9. Hirata M, Tanaka S, Oka S, et al. Evaluation of microvessels in colorectal tumors by narrow band imaging magnification. Gastrointest Endosc. 2007;66:945-952.

10. Hirata M, Tanaka S, Oka S, et al. Magnifying endoscopy with narrow band imaging for diagnosis of colorectal tumors. Gastrointest Endosc. 2007;65:988-995.

11. Chiu HM, Chang CY, Chen CC, et al. A prospective comparative study of narrow-band imaging, chromoendoscopy, and conventional colonoscopy in the diagnosis of colorectal neoplasia. Gut. 2007;56:373-379.

12. East JE, Suzuki N, Saunders BP. Comparison of magnified pit pattern interpretation with narrow band imaging versus chromoendoscopy for diminutive colonic polyps: a pilot study. Gastrointest Endosc. 2007;66:310-316.

13. Katagiri A, Fu KI, Sano Y, et al. Narrow band imaging with magnifying colonoscopy as diagnostic tool for predicting histology of early colorectal neoplasia. Aliment Pharmacol Ther. 2008;27:1269-1274.

14. Rastogi A, Bansal A, Wani S, et al. Narrow-band imaging colonoscopy - a pilot feasibility study for the detection of polyps and correlation of surface patterns with polyp histologic diagnosis. Gastrointest Endosc. 2008;67:280-286.

15. Wada Y, Kudo SE, Kashida H, et al. Diagnosis of colorectal lesions with the magnifying narrow-band imaging system. Gastrointest Endosc. 2009;70:522-531.

16. Sano Y, Ikematsu H, Fu KI, et al. Meshed capillary vessels by use of narrow-band imaging for differential diagnosis of small colorectal polyps. Gastrointest Endosc. 2009;69:278-283.

17. Konishi K, Kaneko K, Kurahashi T, et al. A comparison of magnifying and nonmagnifying colonoscopy for diagnosis of colorectal polyps: a prospective study. Gastrointest Endosc. 2003;57:48-53.

18. Kudo S, Hirota S, Nakajima T, et al. Colorectal tumours and pit pattern. J Clin Pathol. 1994;47:880-885.

19. Uchihara T, Nakamura A, Nakayama H, et al. Triple immunofluorolabeling with two rabbit polyclonal antibodies and a mouse monoclonal antibody allowing three-dimensional analysis of cotton wool plaques in Alzheimer disease. J Histochem Cytochem. 2003;51:1201-1206.

20. Weidner N, Semple JP, Welch WR, Folkman J. Tumor angiogenesis and metastasis-correlation in invasive breast carcinoma. N Engl J Med. 1991;324:1-8.

21. Cianchi F, Palomba A, Messerini L, et al. Tumor angiogenesis in lymph node-negative rectal cancer: correlation with clinicopathological parameters and prognosis. Ann Surg Oncol. 2002;9: 20-26.

22. Kakeji Y, Yamaguchi S, Yoshida D, et al. Development and assessment of morphologic criteria for diagnosing gastric cancer using confocal endomicroscopy: an ex vivo and in vivo study. Endoscopy. 2006;38:886-890.

23. Konerding MA, Fait E, Gaumann A. 3D microvascular architecture of pre-cancerous lesions and invasive carcinomas of the colon. Br J Cancer. 2001;84:1354-1362.

24. Skinner SA, Frydman GM, O'Brien PE. Microvascular structure of benign and malignant tumors of the colon in humans. Dig Dis Sci. 1995;40:373-384.

25. Horimatsu T, Sano Y, Kaneko K, et al. Relationship between MVD and meshed-capillaries using magnifying NBI colonoscopy in colorectal precursor lesions. Hepatogastroenterology. 2009;56: 372-377.

26. Tanaka S, Hirata M, Oka S. Clinical usefulness of narrow band imaging (NBI) in diagnosis and therapeutic endoscopy for colorectal tumor. Stomach Intestine. 2008;43:881-891. 